Monitoring Rocky Shores 
This page intentionally left blank 


\title{
Monitoring Rocky Shores
}

\author{
Steven N. Murray \\ Richard F. Ambrose \\ Megan N. Dethier
}


University of California Press

Berkeley and Los Angeles, California

University of California Press, Ltd.

London, England

(C) 2006 by The Regents of the Lniversity of California

Library of Congress Cataloging-in-Publication Data

Murray, Steven N. (Steven Nelson), 1944-

Monitoring rocky shores / Steven N. Murray, Richard F. Ambrose, Megan N. Dethier.

p. $\mathrm{cm}$.

Includes bibliographical references.

ISBN 0-520-24728-o (cloth : alk. paper)

1. Intertidal ecology-Research-Methodology.

2. Environmental monitoring-Methodology. I. Ambrose, Richard F. (Richard Francis) II. Dethier, Megan Nichols.

III. Title.

$\mathrm{QH}_{541.5} \cdot \mathrm{S}_{35} \mathrm{M}_{7} 2006$

$577.69^{\prime} 9^{-d} \mathrm{dc2} 2$

2005011949

Manufactured in Canada

$\begin{array}{llllllllll}15 & 14 & 13 & 12 & 11 & 10 & 09 & 08 & 07 & 06 \\ 10 & 9 & 8 & 7 & 6 & 5 & 4 & 3 & 2 & 1\end{array}$

The paper used in this publication meets the minimum requirements of ANSI/NISO Z $39 \cdot 4^{8-1992}$ (R 1997) (Permanence of Paper).

Cover photo: Ochre sea stars feeding on mussels near the Bamfield Marine Station in Barkley Sound, British Columbia. 